\title{
Geistliche Liederdichterinnen? Mechthild von Magdeburg, Mechthild von Hackeborn, Gertrud von Helfta
}

\section{Einleitung}

Die Frage nach dem Gattungsprofil mittelhochdeutscher Liedlyrik wäre ganz sicherlich nicht vollständig gestellt, würde der Bezug auf die weiblichen Vertreterinnen der Zunft fehlen. Das geistliche Lied, und zwar innerhalb der Liederdichtkunst des mittelhochdeutschen Sprachraums dasjenige, das seine formale und inhaltliche Gestalt aus dem christlichen Glaubensmythos und der religiösen Selbstvergewisserung bezieht, ist im Mittelalter (und diese Feststellung wirkt zunächst wie eine Selbstverständlichkeit) eine der vielen Domänen der Männer, die ihr inneres Schauen und religiöses Bestreben zum Ausdruck bringen. Dazwischen nach ähnlichen oder ebenbürtigen Werken von Frauen zu suchen, ist eine diffizile Aufgabe, die letztlich zu der Einsicht führt, dass lediglich sieben bis acht Prozent des auf uns gekommenen geistlichen Liedguts aus der Feder von Dichterinnen stammen (was auch die entsprechende Verteilung der Beiträge in diesem Band nahelegt).

$\mathrm{Ob}$ allerdings die Helftaer Mystikerinnen Mechthild von Magdeburg (um 1207-1282), Mechthild von Hackeborn (1241-1299) und Gertrud von Helfta (,Gertrud die Große‘, 1256-1302) unter gattungstypologischem Aspekt ohne weiteres als ,Dichterinnen geistlichen Liedgutes' bezeichnet werden können, ist noch keineswegs ausgemacht und soll in den folgenden Ausführungen näher untersucht werden.

Wie zu zeigen ist, ergibt sich die Zugehörigkeit der drei genannten Dichterinnen zum Gattungskreis der geistlichen Liederdichter allerdings aus einigen für das mittelalterliche Dichten in Liedform charakteristischen Anhaltspunkten, und zwar sowohl in sprachlich-formaler wie auch in inhaltlich-konzeptioneller Hinsicht. Sie ergibt sich insbesondere aus einem maßgeblichen topographischen Bezugspunkt: Das Wirken aller Mystikerinnen bezieht sich auf Bernhards von Clairvaux Spiritualität, wie sie im Zisterzienserinnenkloster St. Marien in Helfta seit dessen Gründung 1258 gelebt wurde. Dieser bei Mansfeld in Sachsen-Anhalt gelegene Ort war zugleich der historische Lebensmittelpunkt und Hauptwirkungsort der drei Dichterinnen. Das provoziert zu der Frage nach Verwandt- 
schaften in der christlich-mystischen Dichtungstradition des Liedes. Hierzu einige vorläufige Beobachtungen.

Gertrud von Helfta, die aufgrund ihres Wirkens als ,Gertrud die Große‘ verehrt wird, ist als Jüngste in den Orden eingetreten. Nach ihrer im Alter von fünf Jahren begonnenen Ausbildung in der Klosterschule trat sie zum frühestmöglichen Zeitpunkt das Noviziat an. Der Grad ihres Kenntniserwerbs in theologischen und humanwissenschaftlichen Gebieten sowie den artes liberales wird als ungewöhnlich umfangreich und profund beschrieben. Doch wegen einer spirituelle Krise, die sie im Alter von 26 Jahren durchlebte, ließ sie sich von der rein intellektuellen Kontemplation abwenden und ganz einem tief empfundenen christlichen Mystizismus widmen. Ihre literarische Tätigkeit gipfelt in den Hauptwerken Exercitia spiritualia und Legatus divinae pietatis, die, zum großen Teil von den Mitschwestern niedergeschrieben, als Diktate oder aufgefangene Formulierungen von Eingebungen zu verstehen sind. Da sie auch lange den klösterlichen Chor leitete, ist es nicht verwunderlich, dass die Musik und darin insbesondere der Gesang und die menschliche Stimme einen großen Teil der Motive bereitstellen, auf die sich Gertrud immer wieder bezieht.

Auch für Mechthilds von Hackeborn Leben und Werk spielte Musik eine entscheidende Rolle. Als jüngere Schwester des damaligen Konventsoberhauptes Gertrud (nicht zu verwechseln mit der o.g. Gertrud der Großen) kam sie - nach einem Besuch ihrer Schwester in Helfta überaus beeindruckt, aber gegen den elterlichen Willen - bereits mit sieben Jahren zum Konvent. Ihr Gesang und ihre Stimme trugen ihr den Beinamen einer ,Nachtigall Gottes' ein. So wird sie früh der Liedform begegnet sein. Auf sie ist der Liber specialis gratiae zurückzuführen, der wohl vor allem dadurch entstehen konnte, da Gertrud und mindestens eine weitere Mitschwester Mechthilds Gebete und Eingebungen, ihre Gedanken und Visionen niederschrieben und so für die Nachwelt erhielten. Ihre rein subjektive Spiritualität und Mystizität stehen wie bei Mechthild von Magdeburg in der Tradition Bernhards von Clairvaux.

Mechthild von Magdeburg, die (ab ca. 1270 und ohne Kanonisierung) bis zu ihrem Tod 1282 als Mitschwester der beiden vorgenannten Mystikerinnen ebenfalls in Helfta lebte, nimmt zwischen diesen und den männlichen Verfassern geistlichen Liedgutes insofern eine Sonderstellung ein, als sie sich der mittelniederdeutschen Sprache bediente. Als Begine war sie zwar nicht einem Kloster verpflichtet, doch war ihr von ihrem Beichtvater angeraten worden, ihre vielfachen mystischen Erfahrungen niederzuschreiben, die dann in dem Werk Vom fließenden Licht der Gottheit ihren Niederschlag fanden. Mit ihrem während des Lebens als Begine begonnenen Wüten gegen ihre eigene Leiblichkeit, daneben ihrer hohen Sprach- und Dichtkunst sowie dem daraus entstandenen, verschiedenste Textgattungen umfassenden Werk gilt sie spätestens seit dem Aufkommen 
einer sog. ,feministischen Mediävistik‘ im zwanzigsten Jahrhundert als bedeutende Vertreterin eines von Frauen getragenen Mystizismus und von Heilserwartungen geprägten Christentums. ${ }^{1}$ Das Werk wendet sich in innigsten Liebesbekundungen und höchster religiöser, individuell geprägter Inbrunst an die göttlichen Kräfte und Verheißungen, vor denen die irdischen Leiden und Nöte verblassen. Sublimierte, auf den unerreichbaren Liebesakt mit dem göttlichen Geliebten (Jesus) gerichtete sexuelle Phantasien lassen das Werk dazu als ein Beispiel hocherotischer Dichtung wirken.

Damit ist zugleich die Frage aufzuwerfen, inwieweit Mechthilds Heils- und Erlösungsphantasien einer bestimmten Gattung zuzuordnen sind. So wird das Fließende Licht sicher nicht zu Unrecht der mittelhochdeutschen Gesangslyrik, und zwar darin der Minnelyrik als der größten Gruppe subsumiert. Doch wesentlicher ist die Beobachtung, wie Mechthilds Dichtkunst die Minne in Jenseitserwartung transformiert, also das sinnliche Erlebnis (etwa einer Liebesnacht mit Jesus) zu inbrünstigem Ewigkeitsverlangen werden lässt. Das Fließende Licht nimmt damit innerhalb der mittelalterlichen Liebeslieddichtung eine herausragende Stellung ein. Es bezieht sich einerseits sozialethisch und kulturell auf die Konzeptionen des zwölften und dreizehnten Jahrhunderts, lässt aber die durchaus asketischeren Ansätze der vorangegangenen Jahrhunderte noch durchscheinen. In der ,Hochzeitskammer des Jenseits' sind diese Sehnsuchtsorte vereint, Zeilen wie „Ich stürbe gern aus Minne“ (1942 vertont von Johann Nepomuk David) beweisen die Versuche, Hingabe und Verzicht, (ungelebtes) sexuelles Verlangen und Jenseitsstreben zu vereinigen. Das Werk geht damit und in vielen weiteren Teilen deutlich über die Tradition geistlicher bzw. liturgischer Texte hinaus und schafft einen eigenen Wirkungsraum.

Wenn man überhaupt von so etwas wie einer ,Norm` sprechen möchte, so zeigt sich, dass ,geistliche Dichtung' durchaus von Form und Inhalt her an kirchliche und liturgische Anforderungen oder Vorgaben gebunden ist. Diese beginnen sich nun aber zugunsten einer Lieddichtungsform zu verändern oder aufzulösen, die in der Nachfolge der von Bernhard von Clairvaux formulierten Prototypen steht und vor allem von den oben genannten Protagonistinnen im Helftaer Kloster weiterentwickelt wurde.

Dabei liegen die Veränderungen auf der Hand, denn die theologisch-ontologische Glücksverheißung im Jenseits (im Sinne eines Dogmas) wird im Fließenden Licht zu einem das rein theoretische Glaubenskonstrukt überstrahlenden inneren Erlebnisraum einer einzelnen Person, die selbst das Zentrum der geistig-

1 Es sei in diesem Zusammenhang verwiesen auf die Arbeiten von Ingrid Bennewitz, 1989, 1993 (siehe Literaturverzeichnis). 
mystischen Schau ist und sich individuell $\mathrm{zu}$ eigenen Glaubensbereichen bekennt. So erscheint es nur zu verständlich, dass das Lied die persönliche, innere Stimme des lyrischen Subjekts erklingen lässt - ein Minnesang im Dienst christlichen Frömmigkeitsempfindens. Dieser Gehalt erneuert und verstärkt den Jenseitsglauben durch das Hinzutreten dessen, was die Begriffe von Emotionalität, Subjektivität und ,persönlicher Betroffenheit‘ in heutigem Sinne bedeuten würden. Die geistliche Liederdichtung übernimmt diese Ausdrucksformen zunehmend.

Die drei Schwestern aus dem Kloster Helfta sind aber nicht die einzigen Frauen, die sich innerhalb des Klosterlebens literarisch äußerten. Durch Anschlüsse und Zusammenlegungen waren im dreizehnten und vierzehnten Jahrhundert viele Frauenklöster zu den Dominikanerinnen, andere, etwa 40 Clarissinnenklöster, zu den Franziskanerinnen gegangen. Nonnenbücher, insbesondere der Dominikanerinnen, gewähren Einblicke in Berichte von Ekstasen und Visionen, z. B. aus dem Kloster Engelthal die Schrift Büchlein von der Gnaden Überlast, in der berichtet wird, dass die Nonnen am ersten Advent nach der Inkorporierung des Klosters (1248) mit ihrer Sangmeisterin, der unmenschlich schon und herrlich singenden Hailrat, nach dem orden (also Latein) gesungen hätten; jedoch am vierten Advent sank sie teutsch und sank so unmenschlich wol, daz man brufet, sie sunge mit engelischer stimme. ${ }^{2}$ Infolge dieses Eindrucks seien die Nonnen von grozer andaht sinnelos geworden und vilen nider als die toten und lagen also biz sie alle wider zu in selber komen. ${ }^{3}$ Ebenso zu erwähnen wäre das Tösser Schwesternbuch mit den Lebensbeschreibungen von neununddreißig Schwestern (um 1340) der Dominikanerin Elsbeth Stagel, die mit dem Mystiker Heinrich Seuse in freundschaftlicher somit persönlicher Verbindung stand. Nicht nur reine Lebensbeschreibungen von Mitschwestern, sondern auch deren Offenbarungen und der Gewinn oder Verlust von göttlichen Gnadenerweisen sind darin thematisiert. ${ }^{4}$

Anders als bei diesen rein erzählenden Texten spielt die Liedform beim Ausdruck mystischen Erlebens eine weit größere Rolle. Als Dichtungen unterschieden sich die liedhaften Texte nicht nur von den Predigten, der Scholastik oder der kanonisierten Mystik, sondern sie blieben Ausdrucksform der Gesinnung in Frauenklöstern, deren Interessen denen der Bettelorden entsprachen und die einen gemeinsamen religiösen Gehalt, einen gemeinsamen geistlichen Kern formulierten.

2 Der Nonne von Engelthal Büchlein von der Genaden Uberlast (ed. Schröder), S. $6 \mathrm{f}$.

3 Koldau, S. 598.

4 Vgl. hierzu den Artikel im Verfasserlexikon von Alois M. Haas. 
Die Stellung der Frau im Mittelalter ist immer wieder Thema von Untersuchungen kultureller und soziologischer Art. Studien zur Differenzierung der Geschlechter legen Fragen danach nahe, welchen Weg die Gesellschaften zwischen Macht, Verehrung und Anerkennung von Frauen auf der einen Seite und der vermeintlichen Misogynie und Unterdrückung des weiblichen Geschlechtes und seiner Leistungen auf der anderen Seite gegangen sind. Das Klosterleben gilt als diejenige Sozialgestalt, in der nicht nur räumliche Geschlechtertrennung, sondern auch völlige Abstinenz im Zölibat als geradezu religiös geboten erscheint und damit als dessen charakteristischster Kern eines solchen Lebens einzuhalten ist. Daraus ergeben sich je völlig getrennte Erlebensbereiche, die das weibliche Mystikertum begünstigten. Nimmt man zugleich an, dass in allen Gebieten des sozialen, kulturellen und geistigen Lebens Männer dominierten, so rückt die Frage nach den Wirkungsmöglichkeiten von Frauen genauso in den Fokus, was durchaus auch heute noch geschehen mag. Die diesbezüglichen Studien dienen einem unverzichtbaren Vorverständnis der Zusammenhänge, die in diesem Aufsatz auf die Betrachtung der nachgelassenen literarischen Erzeugnisse der drei Mystikerinnen von Helfta bezogen werden sollen. Die Bedeutung des Liedes und der Musik in den Werken der Mystikerinnen und deren Beziehung zu den Liedern, den Hymnen und zur Musik überhaupt sollen dabei gleichermaßen betrachtet werden. ${ }^{5}$

Gibt es ,geistliche Liederdichterinnen‘? So lautet die eingangs gestellte Frage, und sie impliziert die Schwierigkeit einer Einordnung mystischen Sprechens in den Kontext des ,Dichtens“ im Mittelalter. Eine solche Einordnung würde schließlich auch bedeuten, den Frauen die Bezeichnung der ,Dichterin‘ zuzugestehen. Daher soll hier zunächst der semantischen Bestimmung dieser Termini Raum gegeben werden.

\section{Die Frage nach dem Autor}

Die Frage nach ,Autor` und ,Autorschaft' im Mittelalter bzw. in mittelalterlichen Texten wurde bereits auf die verschiedensten Weisen untersucht, und die Diskussion darüber ist noch keineswegs als abgeschlossen $\mathrm{zu}$ betrachten. Fotis Jannidis, Gerhard Lauer, Matías Martínez und Simone Winko haben die wichtigsten Auffassungen und Verwendungsweisen des Autorbegriffs zusammenge-

5 Einen guten Überblick mit grundlegender und weiterführender Literatur bietet neben Koldau z. B. The Cambridge Companion to Medieval Women's Writing (2003). 
stellt. ${ }^{6}$ Ihre Modelle umfassen (1) Inspiration, (2) Kompetenz, (3) Autorität, (4) Individualität, (5) Stil, (6) Intention und (7) Copyright. Im hier betrachteten Kontext der Mystik kann man wohl zunächst davon ausgehen, dass die Kriterien der Inspiration und der Individualität eine wichtige Rolle einnehmen, scheinen sie sich doch auf den ersten Blick gegenseitig auszuschließen. Vergleichbar damit ist wohl die Frage, die bereits Platon in seinem Dialog Ion stellt, ob ein von einem Gott inspirierter Rhapsode ein ,Autor' genannt bzw. als solcher bezeichnet werden könne, wenn er doch ,nur“ durch diesen Gott selbst in der Lage gewesen sei, sein Werk zu verfassen. ${ }^{7}$ Er bezeichnet die Dichter als $\dot{\varepsilon} \rho \mu \eta \tilde{\eta} \varsigma$, als Übersetzer, Interpreten (und nicht nur als „Mittler“, wie Jannidis, Lauer, Martínez, Winko nach der Übersetzung Flashars wiedergeben). ${ }^{8}$ Aber auch dieses Übersetzen, Interpretieren erfolgt, enthusiasmiert durch die Musen, aufgrund von Inspiration seitens einer göttlichen Macht. Insofern spielen der Autor, seine Person und seine Intention keine entscheidende Rolle bei der Interpretation der von ihm geschaffenen Schriften und Texte. Dieses Modell eines poeta vates lässt sich mit der Auffassung des Wirkens der hier in den Blick genommenen Mystikerinnen vergleichen. ${ }^{9}$ Die Frage der Autorschaft ist dem Werk Mechthilds von Magdeburg immanent. So formuliert sie selbst in IV,2: Dis buch ist von gotte komen; Und kurz danach: Got ist selber des min urgúnde, das ich in nie bat mit willen noch mit geren, das er diese ding wo̊lte mir geben die in disem büche sint geschriben. ${ }^{10}$ Und wiederum in III,1: [h] ette es gott vor siben jaren nit mit sunderlicher gabe an minem herzen undervangen, ich swige noch und hette es nie getan. ${ }^{11}$ Sara Poor fasst das Problem hinsichtlich Mechthilds Autorschaft zusammen:

In Mechthild we have a writer who does not sign her own name, whose original text and language are little more than a supposition, whose writings survive only in late medieval copies of translations completed after her death, and whose editors mention her name only in passing if at all, attributing the authorship of the book to God in their prologues. ${ }^{12}$

6 Jannidis, Lauer, Martínez, Winko, S. 1-35. Dort mit weiterer Literatur.

7 Platon (ed. Flashar), S. 18f.

8 Jannidis, Lauer, Martínez, Winko, S. 4.

9 Die Vorstellungen, wie man zum Dichten kommt, sind durchaus kulturspezifisch. Bereits bei Homer - und umso mehr bei späteren Autoren wie z. B. Platon - mag die ,Eingebung der Musen` nur noch Rekurs auf die Tradition sein. Aber z.B. im Rigveda haben die Dichter, die sich auch selbst eher als ,Seher‘, d.h. Verkünder einer göttlichen Offenbarung betrachten, durchaus die Vorstellung einer Inspiration, die über sie kommt, und fürchten sich davor, diese Inspiration zu verlieren, bzw. ihrer nicht mehr teilhaftig zu werden. Dies würde ihnen nämlich die Existenzgrundlage entziehen.

10 Mechthild von Magdeburg (ed. Neumann), S. $109 \mathrm{f}$.

11 Ebd., S. 78.

12 Poor, S. 3. 
Neben Mechthilds Annahme, ein Werkzeug der ihr eingegebenen Worte Gottes zu sein, kann natürlich auch unterstellt werden, dass Zurückhaltung und Bescheidenheit eine Rolle spielen, ganz abgesehen von der Überzeugung, der göttlichen Eingebungen eigentlich unwürdig zu sein. Wichtig hierbei zu erwähnen ist sicherlich auch die in der Forschung immer wieder vertretene Auffassung, dass es Mechthild und den anderen Mystikerinnen auch auf keinem anderen Weg möglich gewesen sei, ihre Schriften überhaupt ohne die Autorität Gottes zu legitimieren. ${ }^{13}$

Damit ergibt sich als zweiter Fragenkomplex, inwieweit sich Mechthild selbst als Frau berechtigt sah, als auctor (und damit auch im Sinne einer auctoritas) aufzutreten und sich aus der Empfindung einer als spezifisch weiblich wahrgenommenen oder zugeschriebenen Unzulänglichkeit zu befreien. Nach Poor spielen Bedenken oder gar Angst vor der als eigenständig erlebten Autorschaft von Frauen eine große Rolle. ${ }^{14}$ Nur dadurch, dass sich Mechthild in Formulierungen der Selbstvergewisserung sicher wurde, ihre Autorschaft auf die wahrgenommene Gotteserfahrung gründen zu können, d. h. ihre eigene Autorität oder Autorschaft zugunsten der absoluten Autorität Gottes negieren zu dürfen, kann sie sich als würdig genug einschätzen, ihre Eingebungen niederzuschreiben. Die Autorität, die Inspiration überhaupt kann nur von einer höheren Macht kommen, wie es am Anfang des Prologs des Legatus von Gertruds Mitschwestern formuliert wird:

Omnium bonorum distributor Spiritus Paraclitus, qui spirat ubi vult, prout vult et quando vult, sicut congruentissimum quaerit secretum aspirandi, sic etiam ad salute plirimorum congruentem ordinat modum aspirate in lucem profendi, ut patet in hac famula Dei. ${ }^{15}$

An dieser Stelle ist ein Rückbezug zu Platons Überlegungen hinsichtlich eines poeta vates möglich - unter diesem Gesichtspunkt darf man durchaus von ,Autorinnen' sprechen, von weiblichen auctores, deren Texte wiedergeben, deuten, vermitteln. Sind sie aber auch ,Dichterinnen‘ im eigentlichen Sinne dieses Begriffes?

Das Wort tihtôn, das eine Lehnübersetzung des lat. Intensivums $\mathrm{zu}$ dicere, dictare, darstellt, hat bei seinem ersten Auftreten in der deutschen Volkssprache

13 Hierzu z. B. Zimmermann (1994) und besonders Jensen und Sohn-Kronthaler (2005).

14 Es ist hierbei aber zu bedenken, dass es kein spezifisches ,Problem` weiblicher Autorinnen, Mystikerinnen etc. war, ihre Autorschaft im Rekurs auf die Autorität Gottes stützen zu wollen. Vgl. z.B. Langer (2004).

15 Gertrud von Helfta, Prolog: ,Der Geist, der alle guten Dinge spendet, der atmet, wo er will, wie er will und wann er will, versucht, seine Inspiration als ein höchst angemessenes Geheimnis zu bewahren; aber zum Heil vieler ordnet er auch eine angemessene Art und Weise an, seine Inspiration zu offenbaren, wie diese Magd Gottes deutlich zeigt‘ (S. 1). 
einen Bezug zur Schriftlichkeit. Bei Otfrid findet sich bereits die Ableitung dihta, ,Dichtung6. ${ }^{16}$ Im zwölften Jahrhundert hat sich dieses Lehnwort im deutschen Wortschatz fest etabliert. Etwas anders sieht es mit dem dazugehörigen Nomen agentis tihter aus. Es ist eine Opposition zu erkennen zwischen den mündlich vortragenden Autoren und solchen, die heute eher als ,Epen` bezeichnete Texte verfassten. Erstere werden gerade im Laufe des dreizehnten Jahrhunderts als singer und letztere eben als tihter bezeichnet. Kurt Gärtner verfolgt die Etablierung des Ausdrucks tihter für den Verfasser anhand von Beispielen u.a. aus König Rother, Herborts von Fritzlar Liet von Troye und dem Wigalois Wirnts von Gravenberg. ${ }^{17}$ Die Bezeichnung tihter steht aber auch zu dieser Zeit noch in Konkurrenz zu der Bezeichnung meister, teilweise werden diese synonym verwendet. Jedoch ist auch eine differenzierte Verwendung zu beobachten, wobei meister eine unspezifische Bezeichnung und tihter einen spezifischen Verfasser meint, wie Gärtner am Beispiel von Johanns von Würzburg Roman Wilhelm von Österreich zeigt: ${ }^{18}$

awe zarter maister clar,

2062

genender Strazburger,

Götfrid ain gůt tihter!

Seit Herbort ist außerdem ein weiteres Synonym zu tihter, nämlich pôête, belegt, das im Weiteren den Begriff des tihters verdrängen wird. Trotz der Belege muss auch Gärtner feststellen, dass das Nomen agentis zu dem sich explizit auf das literarische Schaffen beziehenden tihton kein häufig gebrauchtes Wort im Mittelhochdeutschen ist und sich gegen das ungleich häufiger in diesem Zusammenhang gebrauchte meister zunächst nicht so recht durchzusetzen vermochte:

Der Inhalt bzw. der Begriff ,Autor‘ wurde also im Mittelhochdeutschen - jedenfalls im Bereich der Epik - wesentlich ungenauer ausgedrückt als das im Neuhochdeutschen der Fall ist. Es gab möglicherweise kein Bedürfnis, Autor und Autorschaft so differenziert zu bezeichnen, wie wir das heute tun. ${ }^{19}$

Möchte man dem Titel des vorliegenden Tagungsbandes, der sich auf geistliche Liederdichter bezieht, und der in diesem Aufsatz gestellten Frage nach den Liederdichterinnen, also weiblichen Autoren, gerecht werden, so ist darauf hinzuweisen, dass dieses Kompositum eine spezifische Gruppe von Dichtern betont,

16 Shimbo, S. 30.

17 Gärtner, S. 41-46.

18 Vgl. Gärtner, S. 44.

19 Ebd., S. 45. 
nämlich die Verfasser von Liedern. So sind die Texte der drei Helftaschwestern nun darauf zu untersuchen, in welchem Verhältnis sie zu den in ihren Werken enthaltenen Liedern stehen. Dabei wird unter ,Lied' und im speziellen unter ,geistlichem Lied“ ,jedes Lied [verstanden], das einen geistlichen Inhalt hat, gleichgültig, ob es ein einzelner vortrug oder ob es von der Gemeinde gesungen wurde. “20

Ein - wie bereits erwähnt - wesentlicher Unterschied bei der Behandlung der Werke Mechthilds von Magdeburg und der anderen beiden Helftaschwestern ist hierbei die Tatsache, dass Mechthild ihr Fließendes Licht in der Volkssprache verfasste und nicht wie Gertrud und Mechthild von Hackeborn auf Latein. Dabei soll nicht die Liturgizität der Lieder, wie sie etwa die Studien Johannes Janotas ${ }^{21}$ zum deutschen geistlichen Lied in den Fokus rücken, thematisiert werden, sondern die Frage, ob man in den Werken der drei Mystikerinnen überhaupt von ,Liedern' sprechen kann, um dann in einem zweiten Schritt die Überlegungen zur Autorschaft mit dem eventuell aufgefundenen tatsächlichen Liedgut zu verbinden und damit die Titelfrage eventuell zustimmend beantworten zu können.

\section{Die Rolle der Musik in den Schriften der Mystikerinnen}

Nu gebristet mir túsches, des latines kan ich nit - an einer Stelle in ihrem Werk sieht sich Mechthild von Magdeburg an einer Grenze stehen. ${ }^{22}$ Es ist eine selbst empfundene sprachliche Grenze; sie steht nach eigener Aussage vor dem Unsagbaren, wenn sie versucht, ihre Erlebnisse inneren und äußeren Schauens, ihres mystischen Denkens in Worte zu fassen. Doch diese Beobachtung teilt sie mit den beiden anderen Mystikerinnen, die im Gegensatz zu ihr in Latein schrieben, denn auch Gertrud und Mechthild von Hackeborn stoßen an die Grenzen des Sagbaren.

Zur Bewältigung dieser Hürden wenden sich die drei Mystikerinnen den übersprachlichen Ausdrucksformen der Musik und den damit verbundenen Begrifflichkeiten zu. Sie tun dies durchaus unter Rückgriff auf die Tradition, indem sie sich des Mittels der figurata locutio, also einer im weitesten Sinne ,bildlichen“ Rede bedienen.

Obwohl ihre Bekanntheit und Beliebtheit zu jener Zeit von ganz unterschiedlicher Art waren, haben alle drei doch durch das Wirken und Leben im

20 Rupprich, Heger, S. 191.

21 Siehe Literaturverzeichnis.

22 Mechtild von Magdeburg, II,3, (ed. Neumann), S. 40. 
Kloster Helfta ein verbindendes Moment. Dieser Hintergrund schwingt auch in ihren Werken und auch - was hier interessiert - in ihrer Verwendung von Elementen der Musik mit. Dabei lassen sich vergleichbare Anlässe und Zwecke des Auftretens der Musik und des Gesanges ausmachen. Nicht nur sind Musik und Gesang als wichtige Elemente in der Bibel zu finden - wie die Psalmen und das Hohelied, die ja bereits dem Namen nach ,musikalisch“ aufzufassen sind -, sondern auch die Beziehungen zwischen Gott und den Menschen werden an vielen Stellen mit Musik oder Gesang zum Ausdruck gebracht. Man denke etwa an Klage- oder Preislieder oder die ,himmlische Musik‘, die seit dem Buch Henoch geradezu topisch immer wieder anzutreffen ist. ${ }^{23}$ Daher verwundert es kaum, dass auch in den Werken der Mystikerinnen eher allgemeinere Verweise auf Gesang, der vor allem das Lob Gottes zum Ausdruck bringt, zu finden sind. Daneben ist es vor allem die Rolle der Musik, die in den Visionen der drei Helftaschwestern und in Bezug zu Leiderfahrungen wie Krankheit bedeutsam wird. So wird im dritten Buch des Legatus geschildert, wie sehr Gertrud von der Schönheit des Gesangs und der Melodie bewegt wird:

In festo quodam, dum vitio capitis in cantu impediretur, requisivit a Domino, cur saepius in festis sineret hoc ei evenire; et super hoc tale accepit responsum: ,Ne forte per delectationem modulationis elevata, gratiae meae minus idonea inveniaris.‘ Et illa: ,Gratia tua, Domine, posset in me hunc casum praecavere.' Et Dominus: ,Ad meliorem profectum cedit homini, quod sibi occasio casus tollitur per depressionem gravaminis: quia inde duplex illi meritum crescit, scilicet patientiae et humilitatis. ${ }^{24}$

Es lassen sich noch viele weitere Beispiele für Gertruds und Mechthilds von Hackeborn Umgang mit Gesang, Musik und Musikinstrumenten finden, deren offensichtlichster, häufigster oder vielleicht auch ausschließlicher Zweck darin besteht, Gott zu ehren und zu preisen. Selbst die rein praktische Seite, die Beschreibungen der Verwendung von Instrumenten, dient diesem Ziel. Häufig beginnen die Visionen der genannten Mystikerinnen auch mit einem Lied und mit Gesang, oder sie sind Rahmen oder Teil des musikalischen Geschehens. Lob-

23 Vgl. Nieden, S. 26.

24 Gertrud von Helfta, III, 30: ,Eines Tages, als sie durch starke Kopfschmerzen am Singen gehindert wurde, erfragte sie vom Herrn, warum er das so oft an einem Festtag mit ihr geschehen lasse. Sie erhielt diese Antwort: ,Damit du nicht einmal von dem Vergnügen, die heilige Melodie zu singen, mitgerissen und weniger empfänglich für meine Gnade wirst.' Und sie sagte: ,Eure Gnade, Herr, kann mich davor beschützen. ' Der Herr wieder: ,Aber es ist sicher besser für einen Menschen, wenn eine Gelegenheit, in die Sünde zu fallen, durch ein Leiden oder eine Notlage hinfortgenommen wird. Das bringt ihm ein zweifaches Wachstum der Verdienste, denn er erhöht sowohl die Geduld als auch die Demut' (Bd. I, S.185-186). Vgl. zu dieser Stelle auch Savage, S. 81. 
preisungen und Gebete an Gott werden in musikalisch ausgekleideter Form vorgetragen. Musik verbindet die Betenden oder Lobsingenden eher und intensiver mit Gott, als es bloß gesprochene oder gedanklich formulierte Worte erreichen könnten. Musik ist die Kraft, in der auch Antworten, Bestätigungen und Bekräftigungen gesucht und gefunden werden. Ein Beispiel findet sich im zweiten Buch des Liber specialis gratiae, wo sich an einer Stelle das Herz Christi Mechthild in Form einer zehnsaitigen Harfe offenbart, während sie im Chor singt. Sie versteht, dass sie mit dem Zupfen des Psalters die entsprechenden Saiten der Harfe Christi zupfte: $:^{25}$

Exhinc desiderabat ut omnes in coelo et in terra participes Dei gratiae efficierentur. Et accipiens manum Domini, adeo magnam crucem fecit ut videretur sibi coelum et terram inde repleri. Et ex hoc Gaudium coelestium augmentabatur, reis venia, tristibus solamen, justis fortitude et perserverantia dabatur, purgandisque animabus absolution et poenarum alleviation reddebatur. ${ }^{26}$

Daneben stützt Musik auch im pädagogischen Kontext, etwa wenn Hymnen in Form der lectio divina behandelt werden oder wenn Bedeutung und Bildsprache durch Gesänge herausgehoben werden sollen. Musikinstrumente erscheinen so auch als Gegenstände zur Erzeugung von schönen Klängen und Harmonien, die die himmlischen Landschaften zu symbolisieren vermögen.

Wie Gertrud und Mechthild von Hackeborn hat auch Mechthild von Magdeburg in ihrem Werk Das fließende Licht der Gottheit viele Ideen bezüglich der Musik, z. B. die Bedeutung von Gesängen für Visionen und die Messe sowie Verweise auf Bilder und Metaphern von Musikinstrumenten, übernommen oder eigenständig entwickelt. Auch hier muss natürlich berücksichtigt werden, dass Mechthild von Magdeburg sich nicht der lateinischen, sondern der deutschen (im verschollenen Original mittelniederdeutschen) Volkssprache bedient hat. Es ist davon nur eine lateinische Übersetzung (wahrscheinlich ihres Beichtvaters Heinrichs von Halle) und eine oberdeutsche Übertragung erhalten, die vermutlich durch den Basler Weltpriester Heinrich von Nördlingen fast hundert Jahre nach Mechthilds Tod aufgezeichnet und verbreitet wurde. Die Handschrift liegt heute

25 Johnson, S. 183: „[...] she was plucking the corresponding strings of Christ’s harp.“

26 Mechthild von Hackeborn, II, 35: ,Sie wünschte, dass alle Seelen im Himmel und auf Erden an dieser göttlichen Gnade teilhaben würden. Und damit nahm sie die Hand unseres Herrn und machte sich so groß, dass es schien, als würden Himmel und Erde vollständig verbunden sein. Und damit wurde die Freude der himmlischen Geister erhöht, die Vergebung wurde denen gegeben, die schuldig waren. Trost für die, die traurig waren. Kraft und Ausdauer für die Gerechten, während den Seelen im Fegefeuer Absolution und Linderung von Schmerzen gewährt wurde. (Bd. II, S. 182-183). 
vollständig erhalten in der Stiftsbibliothek Einsiedeln vor (Cod. 277 [1014]), woraus auch die hier angeführten Beispiele stammen. Die Musik dient - wie wohl auch nicht anders zu erwarten - der Lobpreisung Gottes.

Wenn der Musik wie bei den anderen beiden Helftaschwestern die Hauptaufgabe zukommt, Lob und Preis Gottes zum Ausdruck zu bringen, dann singen, wie Savage formuliert, verschiedene Wesen im Universum ihre eigenen einzigartigen Lieder: „Not only is most singing in the Light centered around praise, but different beings in the universe (humans, angels, members of the Trinity) sing their own unique songs. “27 Ein schönes Beispiel findet sich im fünften Buch des Fließenden Lichts:

Eya, nu hơre wie dú helige drivaltekeit sich selber lobet mit ir unbeginlicher wisheit und mit ir endelosen gůti und mit ir ewigen warheit und mit ir ganzen ewekeit. Nu hơre die allersůssosten, die allerho̊hsten, die allerwuneklichosten stimme, wie dú helige drivaltekeit in ir selben singet mit einer ganzen stimme, da aller heligen sůssen stimmen usgevlossen sint, die ie gesungen wurdent in himmelriche und im ertriche und noch so̊llent ewekliche. Des vatter stimme sprichet ime lobesange: „Ich bin ein usvliessende brunne, den nieman mag verstoppfen, aber der mensche mag villihte sin herze selber mit eime unnútzen gedank verstoppfen, das dú ungerủewige gotheit, dú iemer mere arbeitet ane arbeit, nit in sin sele mag vliessen.“ Der sun singet alsus: „Ich bin ein widerkomende richtům, den nieman behalten mag, wan alleine dú miltekeit, dú ie gevlos und iemer gevliessen sol von gotte, die kumt alles wider mit sime sune.“ Der helig geist singet dis lob: „Ich bin ein unúberwunden kraft der warheit, das vindet man an dem menschen, der loblich mit gotte bestet swas in angat." 28

Dieses Beispiel ist aus verschiedenen Gründen bemerkenswert. Der Mensch lobt nicht nur die Dreifaltigkeit, sondern reflektiert auch über sich selbst. Darüber hinaus wird die Kommunikation zwischen den verschiedenen Teilen der Göttlichkeit nicht durch gesprochene Worte, sondern durch Musik erreicht. Diese drei Lieder sind wiederum eng mit der Funktion jeder einzelnen Person der Dreifaltigkeit verbunden; es ist fast so, als ob das Lied und die Natur von Vater, Sohn und Heiligem Geist ein und dasselbe wären. Insbesondere die Bilder der Lieder des Vaters und des Sohnes kehren gewissermaßen ,fließend‘ zu einem der Hauptthemen von Mechthilds Werk zurück: In einem Verschwimmen von Sinnen und materiellen wie immateriellen Objekten können Musik, Wasser und Gottes Liebe in die aufnahmebereite Seele fließen.

Diese nur mit kurzen Beispielen unterlegte Betrachtung und Analyse der Texte sollte nachweisen, dass den drei Mystikerinnen die Musik nicht nur als Teil

27 Savage, S. $61 \mathrm{f}$.

28 Mechthild von Magdeburg (ed. Neumann), S. $185 \mathrm{f}$. 
ihres religiösen Verständnishorizonts am Herzen lag, sondern dass die stete Bezugnahme auf Musik eine universale Hierarchie verdeutlicht, die sich auf dem Prinzip der unerschöpflichen Himmelsliebe gründet.

In den Schriften der drei Helftamystikerinnen gibt es viele Gemeinsamkeiten hinsichtlich der Beschreibung von Musikelementen: Die Musik und insbesondere der liturgische Gesang dienen oft als Initialzündung für visionäre Erfahrungen. Während der Gesang das prominenteste und wertvollste Beispiel für Musik ist, treten auch Instrumente, insbesondere Streichinstrumente auf, entweder aus ästhetischen oder aus symbolischen Gründen. Beim Singen werden manchmal die Worte eines Gesangs besonders betont. Zum Beispiel, wenn Gertrud sich trotz Krankheit bemüht, einige Gebete zum Wohle einer Schwester zu wiederholen:

[I]nter Missam dum devotius intenderet Domino, recognovit animam suam valde mirandis ornatibus, et miro modo coruscantibus gemmis pretiosis honorifice decoratam. Intellexitque divinitus instructa, se talem ornatum inde meruisse, quod in charitate humiliter cum juniori iterato legerat partem Matutinarum ante lectam, unde et tot ornatibus præfulgebat, quod verba relegerat. ${ }^{29}$

Die Worte und die Melodie sind also gleichermaßen von Bedeutung. Instrumentalmusik ist genauso bewegend wie Gesang. Dieser erscheint jedoch eher in den Funktionen der Bezeichnung von Schönheit oder der Darstellung der Liebe, die Gott und die Mystiker füreinander haben. So spricht Gott zu Mechthild von Magdeburg im Fließenden Licht (II, 3):

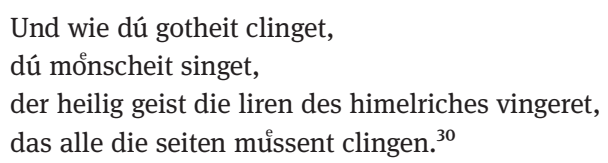

Musik verbindet also sogar alle Ebenen der Schöpfung. Nicht nur die Chöre der Engel und die auferstandenen Heiligen im Himmel richten lobpreisende Lieder an Gott, wie in zahlreichen Visionen gezeigt wird, die Messe dient auch als verbindendes Element zwischen den Bewohnern des Himmels und der Erde. Hier ist auch zu erkennen, was Mechthild von Magdeburg in Bezug auf die Frage, ob sie

29 Gertrud von Helfta, III, 61: ,Während der Messe, als sie noch frommer zum Herrn betete, schien es ihr, dass sie ihre Seele sehr wunderbar mit kostbaren Juwelen geschmückt und mit einem wunderbaren Glanz erstrahlt sah. Göttlich gelehrt verstand sie, dass sie diese Zierde verdient hatte, weil sie demütig und wohltätig mit der jüngeren Nonne den Teil der Matutin, den sie zuvor rezitiert, erneut rezitiert hatte, und dass die leuchtenden Ornamente ebenso zahlreich waren wie die Worte, die sie so wiederholt hatte' (Bd. I, S. 234).

30 Mechthild von Magdeburg (ed. Neumann), S. 40. 
als Dichterin zu bezeichnen sei, von den anderen beiden Helftaschwestern unterscheidet: Während sich Gertrud und Mechthild von Hackeborn auf bereits vorhandenes Hymnenmaterial beziehen, dichtet Mechthild von Magdeburg in ihrem Werk selbst. Wenn man den Begriff des Liedes so weit fasst wie in dem oben genannten Zitat, dass also ein geistliches Lied dadurch gekennzeichnet sei, dass es einen geistlichen Inhalt habe, und zwar unabhängig von seiner Gebrauchssituation, dann ist es m.E. durchaus legitim, Mechthild von Magdeburg eine Liederdichterin zu nennen.

So kann man vielleicht nur Mechthild von Magdeburg als eigentliche Liederdichterin, als creatrix bezeichnen, weil sie selbst Lieder dichtet. Aber alle drei Mystikerinnen haben, wie zu zeigen versucht wurde, jeweils durchaus eine eigene Äußerungs- und Darstellungsform gefunden. Dass sie eigenständig und im Wortsinn aus sich selbst heraus schöpferisch tätig waren, muss ihnen zugestanden werden. Platon behauptet also, dass Gott oder eine göttliche Macht selbst der Dichter sein müsse, sobald sich ein Autor auf entsprechende Eingebungen berufe. Dieser Autor verdiene dann selbstverständlich diesen Ehrentitel nicht, wenn Menschen immer wieder ihre Gedanken als ,Visionen', ,innere Stimmen‘, ,Botschaften aus höheren Sphären', ,Gottes eigene Worte‘ etc. bezeichnen. Davon ausgehend dürfen wir diesen Beglaubigungen selbst oder gerade dann, wenn die Betreffenden sich persönlich fest davon überzeugt zeigen, nicht folgen. Den so gefundenen oder erfundenen Texten, gleich ob gesprochen oder niedergeschrieben, wird so eine Aura unanzweifelbarer Autorität gegeben, der zu widersprechen einer Gotteslästerung gleichkäme.

Bei der Betrachtung der Werke der drei Helftaschwestern befindet man sich in einem interpretatorischen Dilemma. Wenn man sie als ,Dichterinnen“ auffasst, sie sich selbst aber nicht unbedingt als solche begreifen und damit keine kritische Distanz zu ihren Werken zu gewinnen vermögen, welche erforderlich wäre, um zum Beispiel als notwendig empfundene Änderungen, Umgestaltungen, Einfügungen oder Weglassungen durchzuführen, besteht das Problem bei der Auffassung der Unantastbarkeit des Wortes Gottes. Glauben die Frauen aber selbst in tiefster religiöser Überzeugung, Verzückung oder Verklärung, nur Werkzeuge, Stimmen Gottes zu sein, so sind sie dennoch die ,Urheberinnen', die aus heutiger Sicht ein alleiniges Verwertungsrecht an ihren Texten hätten, ein ,Copyright‘, wie es Jannidis u.a. formulieren. ${ }^{31}$

Ein ,Dichter kann also verstanden werden als jemand, dem es gelingt, aus dem Material der Alltagssprache heraus zu einer mehr oder weniger kunstvollen Sprachgestaltung, zu Reimen und Rhythmen, aus seiner Phantasie zur Fiktion zu

31 Siehe oben Fußnote 6. 
gelangen, und als jemand, der dies selbst als eine von ihm bewusst erbrachte, ihm ureigen angehörende Leistung erkennt. Problematisch kann es für die Nachwelt auf den ersten Blick erscheinen, wenn sie aus einem unklaren Entstehungszusammenhang über Menschen urteilen soll, die sich trotz formulierter Werke selbst nicht als Sprachkünstler sehen, sondern ihr intuitives Sprechen, ihr aus ihnen unbewussten Quellen hervorströmendes Schreiben nicht als eigene, sondern bloß ,zugeflossene‘ Leistung auffassen. Was genau die Helftaer Nonnen gehört haben, wenn sie den Liedtexten Stimmen und Instrumentalmusik hinzufügten, kann nur indirekt aus ihren eigenen Berichten geschlossen werden. Doch die Tatsache, dass Musik erklungen ist, dass man Chöre leitete und also auch die Texte der Lieddichterin Mechthild mit Gesang unterlegte, beweist am nachdrücklichsten deren Autorschaft und Qualifikation einer Dichterin, weil sprachliche und musikalische Gestaltung einander bedingen und zu ergänzen vermögen. Zusätzlich geben die Dichterinnen der Musik eine Funktion in ihren Werken, die über das Singen oder Musizieren hinausgeht: Musik und Gesang dienen dem Ausdruck der Verbundenheit mit Gott und zugleich ganz persönlicher, sinnlicher Lobpreisung Gottes.

Entscheidend ist es hierbei nicht so sehr, ob sich die drei Frauen selbst als ,Dichterinnen' sahen, sondern dass sie gewissermaßen objektiviert in der Lage waren, den sie durchfließenden Strom der Inspiration in Worte $\mathrm{zu}$ fassen, zu kanalisieren, und diese auf eine artifizielle, mit Musik verbundene Weise mitzuteilen.

\section{Literaturverzeichnis}

Bennewitz, Ingrid (Hg.): Der frauwen buoch. Versuche zu einer feministischen Mediävistik. Göppingen 1989 (GAG 517).

Bennewitz, Ingrid: Frauenliteratur im Mittelalter oder feministische Mediävistik? Überlegungen zur Entwicklung der geschlechtergeschichtlichen Forschung in der germanistischen Mediävistik der deutschsprachigen Länder. In: ZfdPh 112 (1993) H.3, S. 383-393.

Der Nonne von Engelthal Büchlein von der Genaden Uberlast. Hg. von Karl Schröder. Tübingen 1871 (Bibliothek des Litterarischen Vereins in Stuttgart 108).

Dinshaw, Carolyn (Hg.): The Cambridge Companion to Medieval Women's Writing. Cambridge 2003.

Gärtner, Kurt: Zu den mittelhochdeutschen Bezeichnungen für den Verfasser literarischer Werke. In: Autor und Autorschaft im Mittelalter. Kolloquium Meißen 1995. Hg. von Elizabeth A. Andersen u. a. Tübingen 1998, S. 38-45.

Gertrud von Helfta: Legatus divinae pietatis. In: Revelationes Gertrudianae ac Mechtildianae 1. Sanctae Gertrudis Magnae virginis ordinis sancti Benedicti Legatus divinae pietatis accedit ejusdem Exercitia Spiritualia. Opus ad codicum fidem nunc primum integre editum Solesmensium O.S.B. monachorum cura et opera. Poitiers, Paris 1875.

Haas, Alois M.: Art. ,Tösser Schwesternbuch“ In: VL² 9, Sp. 223-225. 
Jannidis, Fotis, Gerhard Lauer, Matías Martínez, Simone Winko: Rede über den Autor an die Gebildeten unter seinen Verächtern. Historische Modelle und systematische Perspektiven. In: Rückkehr des Autors. Zur Erneuerung eines umstrittenen Begriffs. Hg. von Fotis Jannidis u. a. Tübingen 1999, S. 1-35.

Janota, Johannes: Studien zu Funktion und Typus des deutschen geistlichen Liedes im Mittelalter. München 1968 (MTU 23).

Jensen, Anne, Michaela Sohn-Kronthaler (Hgg.): Formen weiblicher Autorität. Erträge historisch-theologischer Frauenforschung. Wien 2005 (Theologische Frauenforschung in Europa 17).

Johann von Würzburg, Wilhelm von Österreich aus der Gothaer Handschrift hg. von Ernst Regel. Berlin 1906.

Johnson, Ella: The Nightingale of Christ's Redemption Song. Mechthild von Hackerborn's Musical Apostolate. In: Music, Theology, and Justice. Hg. von Michael O'Connor, Hyun-Ah Kim, Christina Labriola. Lanham u. a. 2017.

Koldau, Linda Maria: Frauen-Musik-Kultur: ein Handbuch zum deutschen Sprachgebiet der Frühen Neuzeit. Köln 2005.

Langer, Otto: Christliche Mystik im Mittelalter. Mystik und Rationalisierung - Stationen eines Konflikts. Darmstadt 2004.

Mechthild von Hackeborn: Liber Specialis Gratiae. In: Revelationes Gertrudianae ac Mechthildianae 2. Sanctae Mechthildis virginis ordinis sancti Benedicti Liber specialis gratiae accedit sororis Mechtildis ejusdem ordinis Lux Divinitatis. Opus ad codicum fidem nunc primum integre editum Solesmensium O.S.B. monachorum cura et opera. Poitiers, Paris 1877.

Mechthild von Magdeburg: ,Das fließende Licht der Gottheit'. Hg. von Hans Neumann. Nach der Einsiedler Handschrift in kritischem Vergleich mit der gesamten Überlieferung, 2 Bände. München 1990 (MTU 100).

Nieden, Hans-Jörg: Musik und Theologie. Ökumenische Perspektiven. Berlin 2013.

Platon: Ion. Hg. von Hellmuth Flashar. Griechisch, Deutsch. Stuttgart 1997.

Poor, Sara S.: Mechthild of Magdeburg and Her Book. Gender and the Making of Textual Authority. Pennsylvania 2004.

Rupprich, Hans: Die Deutsche Literatur vom späten Mittelalter bis zum Barock. Erster Teil: Das Ausgehende Mittelalter, Humanismus und Renaissance 1370-1520. 1. Aufl. Neubearb. von Hedwig Heger. München 1994 (Geschichte der deutschen Literatur von den Anfängen bis zur Gegenwart 4,1).

Savage, Christian Gregory: Music and the Writings of the Helfta Mystics. Tallahassee 2012.

Shimbo, Masahiro: Wortindex zu Otfrids Evangelienbuch. Mit alphabetischem und rückläufigem Wortregister. Tübingen 1990 (Indices zur deutschen Literatur 23).

Zimmermann, Béatrice Acklin (Hg.): Denkmodelle von Frauen im Mittelalter. Freiburg/Schweiz 1994 (Dokimion 15). 